International Journal of Clinical Dermatology \& Research (IJCDR)

ISSN 2332-2977

\title{
A Case of Confluent And Reticulated Papillomatosis of Gougerot And Carteaud
}

\author{
Hari Kishan Kumar.Y*, Srivalli P, Ramesh Babu A
}

Case Report

Department of Dermatology, MVJ Medical College \& Research Hospital, Hoskote, Bangalore, Karnataka, India

\section{Abstract}

Confluent and reticulated papillomatosis of Gougerot and Carteaud is a rare dermatosis of unknown etiology, but distinctive acquired icthyosiform dermatosis seen in young adults and characterized by persistent brown, scaly macules, papules, patches and plaques. Lesions tend to be localized predominantly on the neck, upper trunk and axillae where they tend to be confluent and become reticulated towards the periphery. Herein we present a 26 years old male with asymptomatic hyperpigmented and few raised skin lesions over chest, upper back, axillae and upper arms with a characteristic histopathology suggestive of Confluent and reticulated papillomatosis. The importance of differentiating this disease with closely mimicking Pityriasis versicolor and need for prompt treatment of this psychologically upsetting disease in young adults is highlighted.

KeyWords: Confluent Reticulate Papillomatosis

\author{
*Corresponding Author: \\ Hari Kishan Kumar.Y, \\ Associate Professor of Dermatology, \\ MVJ Medical College \& Research Hospital, \\ Hoskote, Bangalore, Karnataka, India. \\ Tel: +9189128607315 \\ E-mail: drkishanyadalla@rediffmail.com
}

Received: August 12, 2013

Accepted: September 26, 2013

Published: September 28, 2013

Citation: Hari Kishan Kumar. Y, Srivalli P, Ramesh Babu A (2013) A Case of Confluent And Reticulated Papillomatosis of Gougerot And Carteaud. Int J Clin Dermatol Res. 1(1), 1-3. doi: http://dx.doi.org/10.19070/2332-2977-130001

Copyright: Hari Kishan Kumar.Y ${ }^{\oplus}$ 2013.This is an open-access article distributed under the terms of the Creative Commons Attribution License, which permits unrestricted use, distribution and reproduction in any medium, provided the original author and source are credited.

\section{Introduction}

Confluent and reticulated papillomatosis (CRP) was described by French dermatologists Gougerot and Carteaud in 1927 [1-3]. The etiology remains obscure, but the main hypotheses that try to explain its onset are keratinization disorder or abnormal response to skin biota microorganisms, which is caused by lipophilic yeast genus Malassezia [2,3]. It is a rare dermatosis which is more frequently found in women, with higher incidence between the ages of 10 and 35 years[3].

\section{Case Report}

A 26 years male presented to our department with complaints of asymptomatic hyperpigmented raised and flat lesions on the upper back, chest, upper abdomen, axillae and flexor aspects of both upper limbs since 2 years. He gives history of aggravation of lesions since 1 year. There was no similar complaints in any other family members. Patient was severely distressed due to the cosmetic disfigurement. The lesions were well defined, brown macules with few slightly elevated, flat-topped, warty papular like lesions on the upper back, shoulders, and intermammary areas which are confluent in the centre and reticulate at the periphery. (Fig.1,2) Microscopic examination of the scrapings in $10 \% \mathrm{KOH}$ did not show any filaments or spores of Malassezia furfur. Routine blood investigations and thyroid profile was normal.

Biopsy of a lesion showed hyperkeratosis and papillomatosis. Dermal papillae projected upwards as finger like projections. Focal acanthosis is seen in the valleys between the elongated papillae with some keratotic material. Dermis showed mild perivascular lymphocytic infiltrate. Biopsy was suggestive of Confluent and reticulated papillomatosis. (Fig.3,4) He was referred to psychiatric consultation for alleviating his distress and was counselled for the same. The patient was started on oral Minocycline $100 \mathrm{mg}$ once daily for 4weeks along with topical tretinoin $0.025 \%$ cream. Patient had good response after completion of the therapy with significant cosmetic improvement and there was no recurrence after 3 months follow-up.

\section{Discussion}

The first case of this disorder was presented by Gougerot and Carteaud in 1927 under the name of Papillomatose pigmentee innominee. In 1932, they classified the cutaneous papillo-matoses into three groups

a. punctate, pigmented and verrucose papillomatosis

b. confluent and reticulated papillomatosis

c. nummular and confluent papillomatosis [3]

Very few cases have been reported from India [8-12]. Most of the reported cases are in African race [4] Occurrence of CRP in members of the same family suggests that it is possibly an inherited disorder [3,5]. As most cases are sporadic, it was suggested that there may be an inherited and an acquired form. Most cases reported were stocky and obese [4]. CRP affects both sexes but two thirds of the patients are women. Disease begins on an aver- 
Figure 1. Multiple hyperpigmented well defined macules with few slightly elevated papule like lesions on chest, shoulders, intermammary areas and upper arms which are confluent in the centre and reticulate at the periphery.

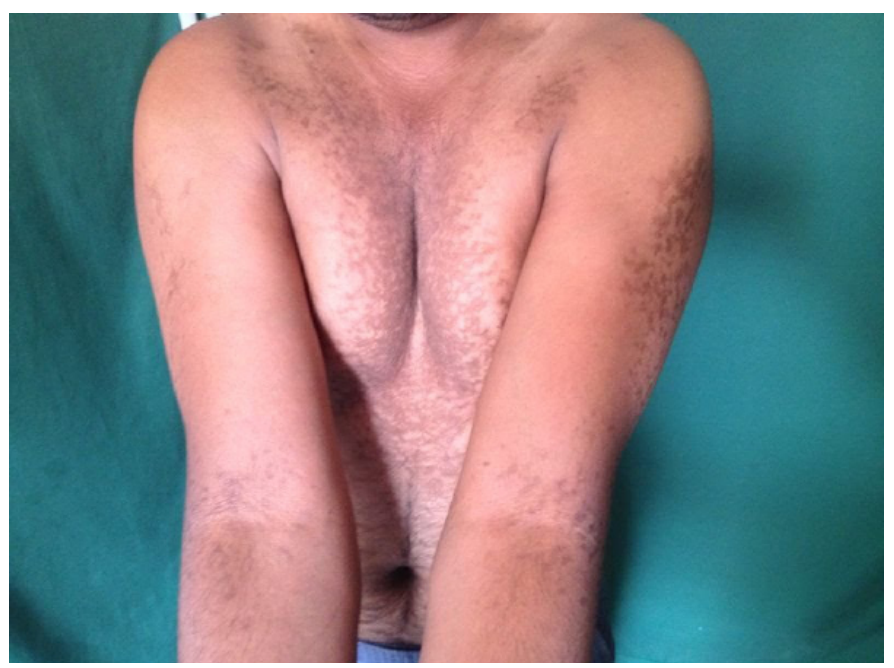

Figure 2. Hyperpigmented macules over the upper back which are confluent in the centre and reticulate at the periphery.

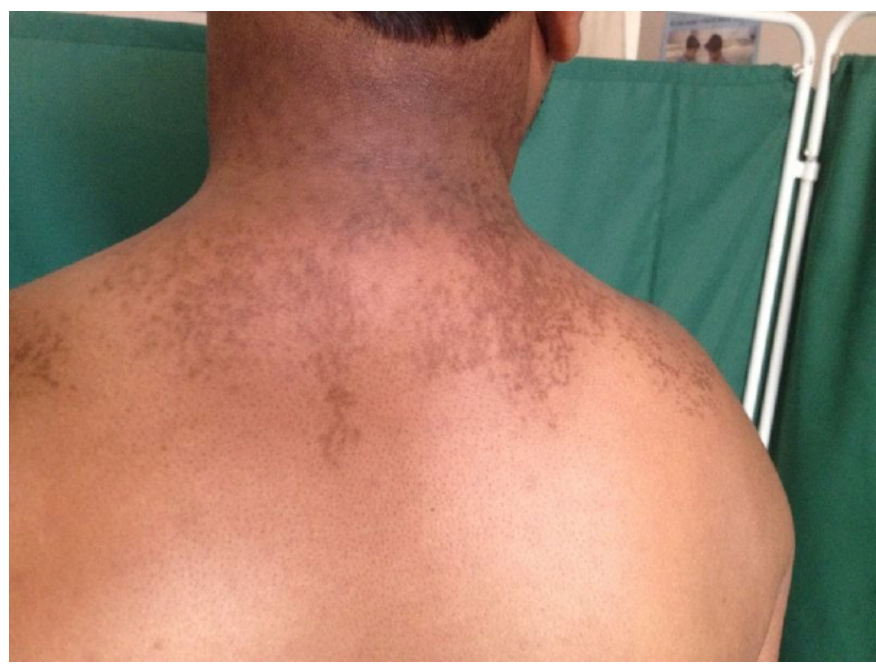

Figure 3. Histopathology of the skin lesion showed epidermis with hyperkeratosis and papillomatosis. Dermal papillae were projected upwards. ( $\mathrm{H}$ and $\mathrm{E} 10 \mathrm{X}$ )

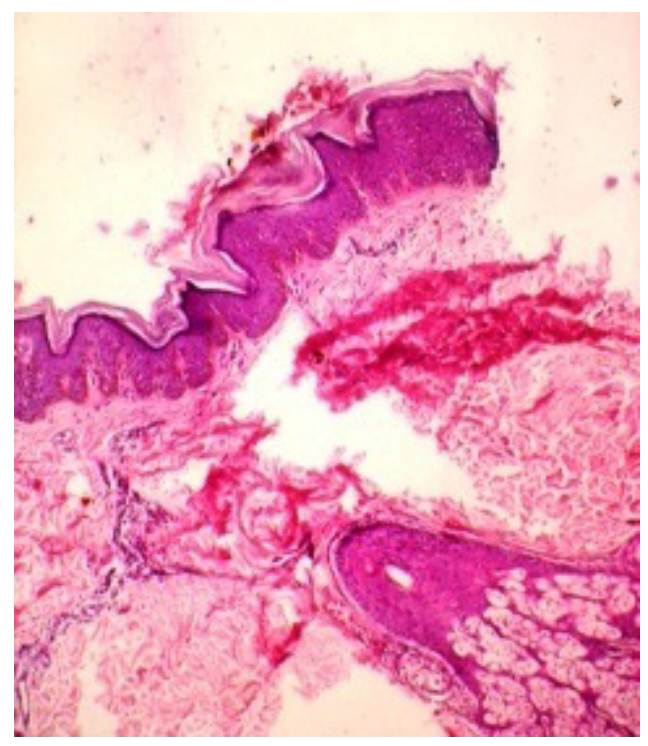

age, in the late teens or early twenties [3]. Our case was an 26years old young adult male, moderately built with no family history with this unusual disease.

Many etiologies have been proposed. Proposed causes include obesity, disturbance of keratinization, exposure to ultraviolet light, endocrine imbalance and infection with Pityrosporum ovale or abnormal host response to Malassezia furfur[1,2]. The onset, distribution and pigmentary disturbance suggest a close similarity to pityriasis versicolor, but examination of the scrapings from the lesions do not demonstrate Pityrosporurn orbiculare (Malassezia furfur). However, treat-ment with local applications effective against P. orbiculare produces clearing of the eruption. Therefore, the underlying defect may be an abnormality of the host 
Figure 4: Histopathology in high power view, shows dermal papillae as finger like projections. Focal acanthosis is seen in valleys between the elongated papillae with some keratotic material. Dermis shows mild perivascular lymphocytic infiltrate. Biopsy features were suggestive of Confluent and reticulated papillomatosis. (H and E 40X)

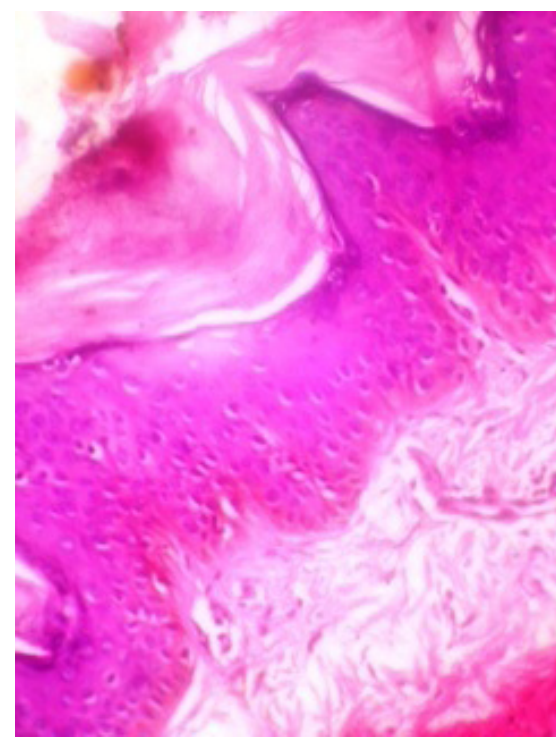

response to colonisation by P. orbiculare[4]. Association of CRP with pseudoatrophoderma colli and acanthosis nigricans has been reported[4]. It was speculated that these three conditions belong to a group of persistent asymptomatic pigmentary disorders $[3,4]$

We implicate abnormal keratinization as the probable etiology in our case among various theories put forth in its pathogenesis as the potassium hydroxide staining of the scale was negative for fungi.

The main differential diagnosis is with Pityriasis versicolor, but others should also be considered: acanthosis nigricans, cutaneous amyloidosis, different keratinization disorders, and some forms of seborrheic dermatitis [1-3] Histopathological examination is not very characteristic and there are subtle affections, correlated with clinical presentation of hyperkeratosis, normally orthokeratotic, irregular papillomatosis, acanthosis predominantly in interpapillary areas and hypogranulosis. There may also be focal atrophy of stratum spinosum, hyperpigmentation of basal layer, without affection to number of melanocytes. On the dermis, there may be chromatophores on the papillary region, discreet mononuclear superficial perivascular infiltrate, mild ectasis of vessels, and small to moderate papillary edema $[1-3,5,6]$. The histopathological differential diagnoses include acanthosis nigricans, variants of seborrheic keratosis, non-inflammation epidermal nevus, DowlingDegos disease and Becker nevus [5,6] Electron microscopy shows increase in transitional cell layer between stratum granulosum and stratum corneum. This fact may define CRP as a disorder of keratinization of skin focal area, without defining whether it would be primary or secondary[5]. Even in our case, histopathology of the skin biopsy showed features that of CRP.

There is no standard therapy for CRP. Minocycline was first used for the treatment of CRP in 1965 by Carteaud. It was found to be highly effective in most of the patients $[4,7,8]$. Perhaps minocycline eradicates an unknown organism involved in predisposing to CRP or helps to suppress an autoimmune phenomenon that might lead to an increase in keratinization[7]. Tetracycline derivatives possess anti-proliferative and anti-inflammatory action and they also inhibit Propionibacterium acnes, collagenase activity and complement activation system[10]. Oral fluconazole $150 \mathrm{mg}$ per week for one month is also effective and also topical 1\% clotrimazole cream. Various other treatment modalities which have been used are topical tretinoin, topical calcipotriol, oral etretinate and isotretinoin and various other oral antibiotics like fusidic acid, erythromycin, clarithromycin. Treatment with oral azithromycin is also successful [12]. There was complete clearance with oral minocycline and topical tretinoin in our patient after 4weeks of treatment with good cosmetic improvement without recurrence at 3 months follow-up. Hence we emphasize on recognizing this unusual disorder which can be extremely distressing and it is imperative that the treatment be effective and swift.

\section{References}

[1]. Judge MR, McLean WHI, Munro CS. Disorders of Keratinization. In: Burns T, Breathnach S, Cox N, Griffiths C, Rook`s textbook of Dermatology. 8th ed. Wiley-Blackwell; 2010.p.19.1-122.

[2]. Fleckman P, DiGiovana JJ. The Ichthyoses. In: Wollf K, Goldsmith LA, Kats S, Gilchrist BA, Paller AS, Leffell DJ. Fitzpatrick`s Dermatology in General Medicine. 7 ed. New York: Mc Graw-Hill; 2008.p.401-424.

[3]. Waisman M. Cutaneous papillomatosis. In: Demis DJ. Clinical Dermatology. Harper and Row, Philadelphia, 1985; Vol 1, Unit 1-42, : pp 1-5.

[4]. Scheinfeld N. Confluent and reticulated papillomatosis: a review of the literature. Am J Clin Dermatol 2006; 7: 305-13.

[5]. Lee SH, Choi EH, Lee WS, Kang WH, Bang D. Confluent and reticulated papillomatosis: a clinical, histopathological, and electron microscopic study. J Dermatol. 1991;18:725-30.

[6]. Berger CM. Clinical pathological challenge: confluent and reticulated papillomatosis of Gougerot and Carteaud. Am J Dermatophatol. 2003;25:17980 .

[7]. Davis MD, Weenig RH, Camilleri MJ. Confluent and reticulate papillomatosis(Gougerot-Carteaud syndrome): a minocycline-responsive dermatosis without evidence for yeast in pathogenesis. A study of 39 patients and a proposal of diagnostic criteria. Br J Dermatol2006; 154:287-93.

[8]. Yesudian P, Kamalam S, Razack A. Confluent and reticulated papillomatosis (Gougerot-Carteaud). An abnormal host reaction to Malassezzia furfur. Acta Derm Venereol 1973; 53:381-4.

[9]. Sardana K, Goel K, Chugh S. Reticulate pigmentary disorders. Indian J Dermatol Venereol Leprol 2013;79:17-29.

[10]. Rao T N, Guruprasad P, Sowjanya CL, Nagasridevi I. Confluent and reticulated papillomatosis: Successful treatment with minocycline. Indian J Dermatol Venereol Leprol 2010;76:725.

[11]. Rajendran N : Confluent and reticulate papilloma 7 tosis, Ind J Dermatol Venereol Leprol, 1989; 55; 51-52.

[12]. Raja Babu KK, Snehal S, Sudha Vani D. Confluent and reticulate papillomatosis: Successful treatment with azithromycin. $\mathrm{Br} J$ Dermatol 2000;142:1252-3. 